\title{
A Review - Design of Area and Power Efficient Digital FIR Filter Based On Faithfully Rounded Truncated 12-Bit Constant
}

\author{
Sonal Gupta \\ M.Tech scholar \\ Lakshmi Narain \\ College of Technology, \\ Bhopal (M.P.)
}

\author{
Umashankar Kurmi \\ Professor \\ Lakshmi Narain \\ College of Technology, \\ Bhopal (M.P.)
}

\begin{abstract}
Finite impulse response (FIR) designs are given exploitation the conception of rounded truncated multipliers 12bit. we have a tendency to think about the optimization of bit width without sacrificing the frequency response and output preciseness. A smallest amount comes to of dissimilar pairs or groups of symbols and residues are often used to code a set of coefficients support on their likelihood and conditional probability of occurrence. This ingenious idea permits the notion of entropy to be applied as a quantitative measure to evaluate the coding density of various compositions of symbols towards a set of coefficients. No uniform constant quantization with correct filter order is projected to attenuate total region expenditure. Multiple invariable multiplication/increase during an exceedingly in a very $\}$ direct FIR structure is implemented exploitation an improved version of truncated multipliers. Multiple constant multiplication/ accumulation during an exceedingly in a very synchronized direct FIR structure are implemented using an improved version of truncated multipliers.
\end{abstract}

\section{Keywords}

Finite impulse response (FIR), multiple constant multiplications (MCM), digital signal processing (DSP)

\section{INTRODUCTION}

\subsection{FIR Filter}

Finite impulse response (FIR) filtering is one among the foremost ordinarily used DSP function. it's achieved by convolving the input file samples with the specified unit impulse response of the filter. The output $\mathrm{Y}$ (n) of an $\mathrm{N}$-tap FIR file add of latest $\mathrm{N}$ input file samples- $\mathrm{N}$

$\mathrm{Y}[\mathrm{n}]=\Sigma \mathrm{A}[\mathrm{i}] . \mathrm{X}[\mathrm{n}-\mathrm{i}] \mathrm{i}=0$

The basic FIR filter is expressed as

$$
Y[n]=\sum_{i=0}^{m-1} a_{i} x[n-i]
$$

In an M-level synchronized system, the number of delay components in any path from input to output is (M-1) greater than that within the same path in the original sequential circuit. Pipelining reduces the critical path, but cause a penalty in terms of an increased latency. Latency is the difference within the accessibility of the primary output information within the pipelined system and therefore the consecutive system. 2 main drawbacks: increase in the number of latches and in system. Finite impulse response (FIR) digital filter is one of the fundamental elements in several digital signal processing(DSP) and communication systems. it's also normally used in many transportable applications with restricted area and power budget.

In case of linear section, the coefficients are either symmetrical or antisymmetric with $a i=a M-i$ or $a i=-a M-i$ There are 2 basic FIR structures, direct type and transposed form, as shown in Fig. 1 for a linear-phase even-order FIR filter. Within the direct type in Fig. 1(a), the multiple constant multiplication (MCM)/accumulation (MCMA) module performs the parallel multiplications of individual delayed signals and respective filter coefficients, followed by accumulation of all the product. Thus, operands of the multipliers in MCMA are delayed input signals $x$ [n - i] and coefficients ai. The weights $\mathrm{A}[\mathrm{i}]$ within the expression are the filter coefficients. the number of taps $(\mathrm{N})$ and therefore the coefficient values are derived thus as to satisfy the desired filter response of pass-band ripple and stop-band attenuation.

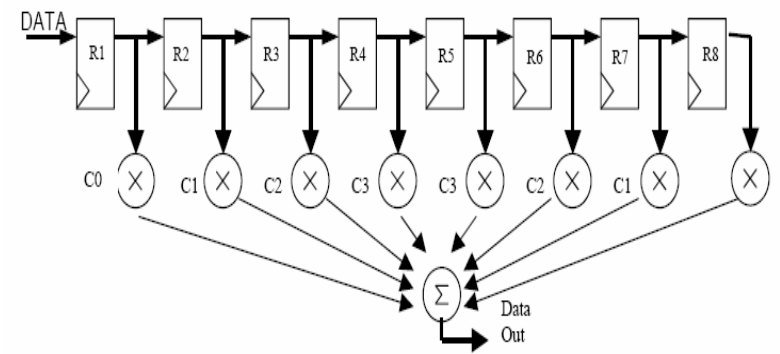

Fig 1: FIR Filter Architecture

Multiplication of 2 numbers generates a product with twice the original bit width. it's usually desirable to truncate the merchandise bits to the required precision to reduce area value, leading to the planning of truncated multipliers or fixed-width multipliers. Fixed-width multipliers, a set of truncated multipliers, compute only $\mathrm{n}$ most important bits (MSBs) of the $2 \mathrm{n}$-bit product for $\mathrm{n} \times \mathrm{n}$ multiplication and use additional correction/compensation circuits to reduce truncation errors. Finite impulse response (FIR) filters are widely utilized in digital signal applications like speech coding, image processing, sampling of multiple systems, etc. though the stability and linear section is secure, the complexness and power consumption of the linear section FIR filter are usually a lot of higher than that of the infinite impulse response (IIR) filter which meets an equivalent magnitude response specification. Therefore, many efforts have been dedicated to the design of low quality and lowpower linear section FIR filters.

There are 2 basic FIR structures, direct type and transposed 
type for a linear-phase even-order FIR filter. within the direct type in the multiple constant multiplication (MCM)/accumulation (MCMA) module performs the concurrent multiplications of individual delayed signals and individual filter coefficients, followed by accumulation of all the product. Thus, the operands of the multipliers in MCMA are delayed input signals $\mathrm{x}[\mathrm{n}-\mathrm{i}]$ and coefficients.

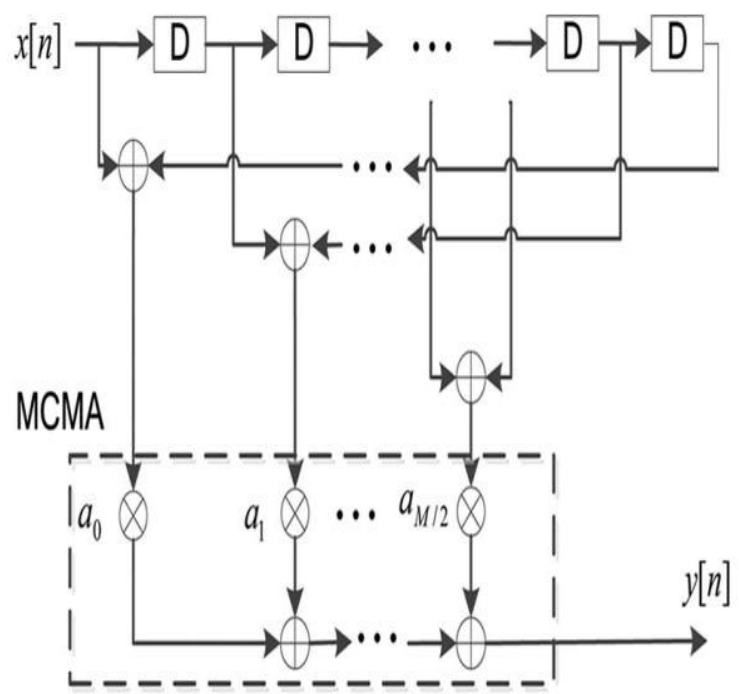

Fig 2: Direct Form of FIR Filter

\section{LITERATURE SURVEY}

M. Peiro. et. al. [1] "Design of high-speed multiplier less filters using a no recursive signed common subexpression algorithm" In this work, a new algorithmic rule referred to as non recursive signed common sub expression elimination (NR-SCSE) is discussed, and a number of function within the area of multiplier less finite-impulse response (FIR) filters are developed. Whereas the recursive utilization of a typical sub expression generates a high logic depth into the digital structure, the NR-SCSE algorithms consent to the designer to surmount this difficulty by using every sub expression once. The paper presents an entire description of the algorithmic rule, and a comparison with 2 alternative well-known options: the graph synthesis, and therefore the classical common sub expression elimination technique. Main results show that the NR-SCSE implementations of many benchmark circuits provide the best relation between occupied area and logic depth regard to the previous values published within the technical literature.

Chip-Hong chang et. al. [2] "Information theoretic Approach to complexness Reduction of FIR Filter Design" this is often author presents a new paradigm of design methodology to reduce the complexness of application-specific finite-impulse response (FIR) digital filters. a new adder graph data structure known as the multiroot binary partition graph (MBPG) is projected for the formulation of the multiple constant multiplication drawback of FIR filter design. The set of coefficients in any fixed point representation is partitioned off into symbols so common sub expression identification and elimination become congruent to information parsing for knowledge compression. A minimum number of various pairs or groups of symbols and residues will be used to code a group of coefficients based on their probability and conditional probability of occurrence. This ingenious conception allows the notion of entropy to be applied as a quantitative measure to evaluate the coding density of different compositions of symbols towards a set of coefficients. The minimal vertex set MBPG synthesized by our planned info theoretical approach leads to direct correspondences between the vertices and adders, and edges and physical interconnections. not like the common sub expression elimination algorithms supported other graph data structures, the symbol-level information carried in every vertex and therefore the graph isomorphism of MBPG promise additional fine-grain optimization in an exceedingly reduced search space. One such optimization that has been exploited in this paper is the shift-inclusive computation rearrangement to minimize $\}$ the width of every two's complement adder to further reduce the implementation cost and therefore the critical path delay of the filter. Experiment results show that the projected algorithmic rule can contribute up to $19.30 \%$ reductions in logic complexness and up to $61.03 \%$ reduction in critical path delay over different minimization ways.

Fei Xu et. al. [3] "Contention Resolution-A New Approach to Versatile Subexpressions Sharing in Multiple Constant Multiplications" Multiple constant multiplications (MCM) are a core operation in several digital signal process applications. during this paper, an efficient generalized contention resolution algorithmic rule (CRA) is projected to eliminate 3 broad classes of reusable common sub expressions in MCM. the concept is to revert a precedential decision of suboptimal common sub expressions by a localized cost function evaluation when there's a conflict between 2 competitive sub expressions. The planned derivatives of the fundamental CRA are versatile in this they're capable of satisfying look for each intra- and inter coefficient sub expressions, in any legitimate composition of horizontal, vertical and oblique sub expressions. because the algorithms expand the common sub expressions to higher-weight only if there are cost saving, the logic depth are often controlled by constrictive the weights of the sub expressions. The variants of CRA follow a crucial tenet of excellent heuristic that significant improvement within the solution quality is attained with increased drawback size but the computational time remains well bounded. Experimental results with each benchmark filters and at random generated coefficient sets are analyzed and compared with a number of well known common sub expression elimination methods to demonstrate the effectiveness and efficiency of our proposed approach.

Hyeong-Ju et. al. [4] "Digital filter synthesis based on an algorithm to generate all minimal signed digit representations" In this the authors propose an algorithmic rule to search out all the minimal signed digit (MSD) illustrations of a constant and present an algorithmic rule to synthesize digital filters supported the MSD representation. The hardware complexity of a digital signal processing system is dependent on the number system used for the implementation. though the canonical signed digit (CSD) illustration is widely utilized, because it is exclusive and guarantees the minimal number of nonzero digits for a continuing, the MSD representation provides multiple illustrations that have an equivalent number of nonzero digits because the CSD representation. The projected filter synthesis algorithmic rule utilizes this redundancy of the MSD representation to make common sub expressions, as many as possible, leading to smaller filters. By applying the proposed algorithmic rule to the hardware synthesis of finite impulse response filters, the authors obtained multiplier factor blocks that are seven-membered smaller than those generated from the CSD representation.

A. Bhuvaneswari et. al. [5] "Low Power Fir Filter Design Using Truncated Multiplier" In this author low-cost finite 
impulse response (FIR) design are given using the concept of faithfully rounded truncated multipliers. we have a tendency to conjointly consider the effective of bit width and hardware resources without sacrificing the frequency response and output signal accuracy. Non-uniform coefficient quantization with proper filter order to reduce the cost of total area. Multiple constant multiplication/accumulation in an exceedingly pipelined direct FIR structure is implemented using an improved version of truncated multipliers. Comparisons with previous FIR filter design approaches show that the projected design succeed the most effective area and power results.

\section{METHOD}

The FIR filter design in this brief adopts the direct form in Fig. 4 where the MCMA module sums up all the products $x[n$ - i].Instead of accumulating individual multiplication for every product, it's additional economical to gather all the PPs into one PPB matrix with carry-save addition to cut back the height of the matrix to 2, followed by a final carry propagation adder. Fig. fourteen illustrates the distinction of individual multiplications and combined matrix. The complements of PPBs are denoted by white circles with over bars. In the faithfully rounded FIR filter realization and it is required that the total error introduce during the arithmetic operations are no larger than one ulp. We modify a recent truncated multiplier design in so that more PPBs may be deleted, leading to smaller space cost. Fig. 4 compares the 2 approaches. within the removal of spare PPBs consists of 3 processes: deletion, truncation, and rounding error. 2 rows of PPBs are set undeletable as a result of they will be removed at the following truncation and rounding. The fault ranges of deletion, truncation, and rounding before and after adding the offset constants.

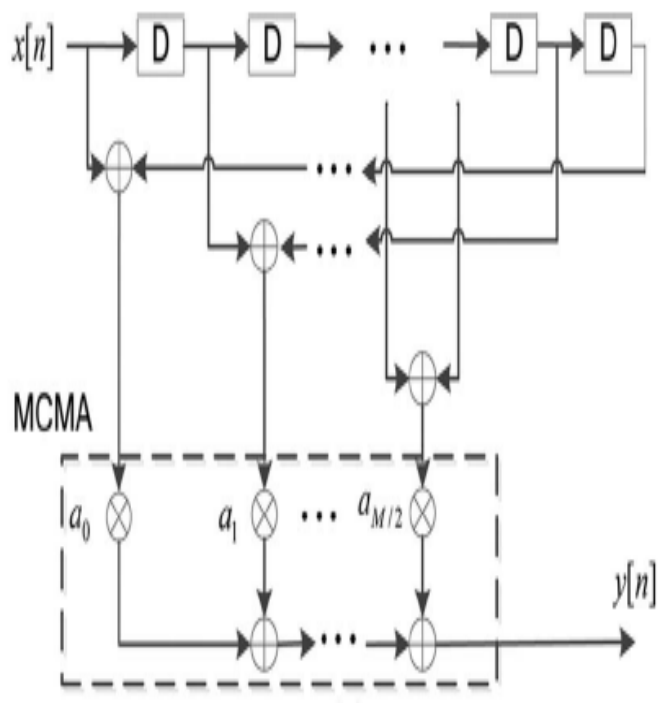

Fig4: Direct form linear phase FIR filter

The gray circles, crossed green circles, and crossed red circles respectively the deleted bits, truncated bits, and rounded bits. Synchronized MCMA with pipeline results in cut back the essential path. Either will increase the clock speed (or sampling speed) or reduces the facility consumption at same speed in a DSP system. Pipelining is reducing the effective essential path by introducing pipelining latches on the essential information path. The pipelined implementation by introducing 2 further latches within the essential path is reduced from $\mathrm{TM}+2 \mathrm{TA}$ to $\mathrm{TM}+\mathrm{TA}$. The schedule of events for this pipelined system. you'll be able to see that, at any time, two consecutive outputs square measure computed in an interleaved manner.

Multiple constant multiplication/accumulation \{in a| during a in associate degree exceedingly| in a very \} direct FIR structure is implemented using an improved version of truncated multipliers. Comparisons with previous FIR style approaches show that the planned styles accomplish the most effective space and power results.

A generic flow of FIR filter style and implementation may be divided into three stages: finding filter order, constant quantization, and hardware optimization. within the first stage, the filter order and also the corresponding coefficients of infinite exactness are determined to satisfy the specification of the frequency response. Then, the coefficients are quantity to finite bit accuracy. Lastly, various optimization approaches like cse are wont to minimize the area cost of hardware implementations. Most previous FIR filter implementations concentrate on the hardware optimization stage. Behind FIR filter operations, the output signals have larger bit width attributable to bit width growth when multiplications. In many practical things, solely partial little bit of the full-precision outputs is required.

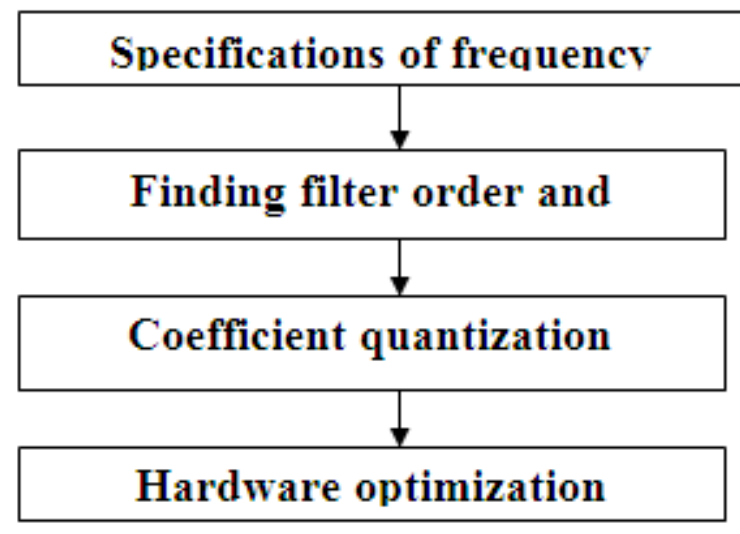

Fig5: Stages of digital FIR filter design and implementation

For example, presumptuous that the input signals of the FIR filter have 12 bits and also the filter coefficients are quantity to ten bits, the bit dimension of the resultant FIR filter output signals is a minimum of twenty-two bits, however we'd want solely the 12 most important bits for consequent process. during this temporary, we tend to adopt the direct FIR structure with MCMA as a result of the realm price of the flipflops within the delay components is smaller compared thereupon of the reversed kind. moreover, we tend to together think about the 3 style stages so as to realize additional economical hardware style with reliably rounded output signals. in contrast to standard uniform quantization of filter coefficients with equal bit dimension, the non uniform quantization technique with probably totally different bit widths is adopted during this temporary.

\section{CONCLUSION}

Abb Low-cost FIR filter designs by jointly considering the improvement of constant bit width and hardware resources in implementations. In proposed work synchronous truncate multiplier factor with delay design to reduce space and delay. Multiplication is main elementary unit of the processer that provides high speed efficient space. Multiplication is one in 
all the basic arithmetic operations and it needs substantially a lot of hardware resources and interval than addition and subtraction. By using a new shortened multiplier factor style by together considering the reduction, deletion, truncation, and rounding of the PP bits. though most previous styles are supported the transposed form, we have a tendency to examine that the direct FIR structure with authentically rounded MCMAT ends up in the smallest amount space cost and delay. In proposed work synchronous truncate multiplier factor with delay design to scale back space and delay.

\section{REFERENCES}

[1.] M. M. P., E. I. B., and L. Wanhammar, "Design of highspeed multiplier less filters using a nonrecursive signed common subexpression algorithm," IEEE Trans. Circuits Syst. II,. Signal Process, no. 3, pp. 196-203.

[2.] C.-H. Chang, J. Chen, and A. P. Vinod, "Information theoretic approach to complexity reduction of FIR filter design," IEEE Trans. Circuits Syst. I, Reg. Papers, vol. 55 , no. 8 , pp. 2310-2321.

[3.] F. Xu, C. H. Chang, and C. C. Jong, "Contention resolution-A new approach to versatile subexpressions sharing in multiple constant multiplications," IEEE Trans. Circuits Syst. I, Reg. Papers, vol. 55, no. 2, pp. $559-571$.

[4.] I.-C. Park and H.-J. Kang, "Digital filter synthesis based on an algorithm to generate all minimal signed digit representations," IEEE Trans. Comput.-Aided Design Integr. Circuits Syst., vol. 21, no. 12, pp. 1525-1529.
[5.] A.Deepika, A.Bhuvaneswari, "Low Power Fir Filter Design Using Truncated Multiplier" International Journal of Engineering Trends and Technology (IJETT) - Volume 10 Number 1 Apr 2014

[6.] Shen Fu Hsiao, Jun Hong Zhang Jian, and Ming Chih Chen,'Low Cost FIR FilterDesigns Based on Faithfully Rounded Truncated Multiple Constant Multiplication/Accumulation,'IEEE transactions on circuits and systems II: Exp.Briefs, vol. 60, No. 5, May 2013

[7.] P. K. Meher, "New approach to look-up-table design and memory-based realization of FIR digital filter," IEEE Trans. Circuits Syst. I, Reg. Papers, vol. 57, no. 3, pp. 592-603, Mar. 2010.

[8.] S. Hwang, G. Han, S. Kang, and J.-S. Kim, "New distributed arithmetic algorithm for low-power FIR filter implementation," IEEE Signal Process. Lett. vol. 11, no. 5, pp. 463-466, May 2004.

[9.] Jiafeng Xie, Jian jun He, and P. K. Meher, ,'Low Latency Systolic Montgomery Multiplier for Finite Field GF(2m) Based on Pentanomials" IEEE Trans. On Very Large Scale Integration (VLSI) Sys., Vol. 21, No. 2, February 2013.

[10.]Ron S. Waters, . and Earl E.Swartzlander,"A Reduced Complexity Wallace Multiplier Reduction”, IEEE Trans. On Computers, . No. 8, August 2010 